\title{
Endovascular versus open elephant trunk completion for extensive aortic disease
}

\author{
Eric E. Roselli, MD, ${ }^{\mathrm{a}}$ Sreekumar Subramanian, MD, ${ }^{\mathrm{b}}$ Zhiyuan Sun, BS, ${ }^{\mathrm{c}}$ Jahanzaib Idrees, MD, ${ }^{\mathrm{a}}$ \\ Edward Nowicki, MD, ${ }^{\mathrm{c}}$ Eugene H. Blackstone, MD, ${ }^{\mathrm{a}, \mathrm{c}}$ Roy K. Greenberg, MD, ${ }^{\mathrm{a}, \mathrm{d}}$ \\ Lars G. Svensson, MD, $\mathrm{PhD},{ }^{\mathrm{a}}$ and Bruce W. Lytle, $\mathrm{MD}^{\mathrm{a}}$
}

Objectives: To compare the outcomes between patients undergoing endovascular (EEC) or open (OEC) approaches to second-stage elephant trunk completion (EC).

Methods: From 1993 to 2010, 225 patients underwent second-stage EC (EEC, $\mathrm{n}=92$; OEC, $\mathrm{n}=133$ ). Propensity matching was performed for a fair comparison.

Results: The EEC patients were older, more likely to have atrial fibrillation, and had a smaller proximal aorta. The 30-day mortality was $6.2 \%(6.5 \%$ EEC vs $6 \%$ OEC, $P=.88)$. No difference was found in bleeding $(8.8 \%)$, stroke $(3 \%)$, renal failure $(4 \%)$, or spinal cord injury $(4 \%)$; however, the OEC patients required tracheostomy more often (10 vs $1, P=.014)$. Survival after second-stage EC at 6 months and 1 and 5 years was $91 \%, 90 \%$, and $77 \%$, respectively. Survival and major morbidity did not differ after matching (44 pairs). However, the EEC group had shorter stays $(9.9 \pm 13$ vs $13 \pm 9$ days, $P<.0001)$ and received less blood $(3 \pm 8$ vs $6 \pm 8 \mathrm{U}$, $P=.0001)$ than did the OEC group. This was maintained after matching. During follow-up, 32 endoleaks (3 type I, 27 type II, 2 type III) occurred; 26 (28\%) EEC and 13 of 76 (17\%) OEC patients underwent reoperation. The approach was not related to the risk of death in either hazard phase, but a larger descending diameter predicted a greater risk in the early phase.

Conclusions: Death and complications occur similarly after OEC or EEC. The early toll might be greater after OEC, at the cost of reintervention for EEC. EEC expands the options to older patients and allows for earlier completion. Second-stage repair should not be delayed, and all patients require lifelong imaging surveillance.

(J Thorac Cardiovasc Surg 2013;146:1408-17)

Patients with extensive thoracic aneurysmal disease present a major surgical challenge. The 2-stage modified elephant trunk approach offers a reasonable solution; however, undergoing both operations is daunting for most patients. In the largest series, $19 \%$ to $60 \%$ of the patients did not return for the second stage and $12 \%$ to $25 \%$ of the patients died between repairs. ${ }^{1-6}$

Endovascular repair of isolated descending aneurysms is less morbid than open repair; however, purely endovascular techniques are not suitable for all patients. Soon after the introduction of stent grafts to treat thoracic aortic disease, hybrid aortic reconstruction combining first-stage elephant

From the Aorta Center and Department of Thoracic and Cardiovascular Surgery, ${ }^{\mathrm{a}}$ Department of Vascular Surgery, ${ }^{\mathrm{d}}$ Heart and Vascular Institute, and Quantitative Health Sciences, ${ }^{\mathrm{c}}$ Cleveland Clinic, Cleveland, Ohio; and University of Arizona Medical Center, ${ }^{\mathrm{b}}$ Tucson, Ariz.

Disclosures: Authors have nothing to disclose with regard to commercial support.

Read at the 36th Annual Meeting of Western Thoracic Surgical Association, Ojai, California, June 25, 2010.

Received for publication June 26, 2010; revisions received July 15, 2013; accepted for publication July 30, 2013; available ahead of print Sept 26, 2013.

Address for reprints: Eric E. Roselli, MD, Aorta Center and Department of Thoracic and Cardiovascular Surgery, Cleveland Clinic, 9500 Euclid Ave, J4-1, Cleveland, OH 44195 (E-mail: roselle@ccf.org).

$0022-5223 / \$ 36.00$

Copyright (c) 2013 by The American Association for Thoracic Surgery

http://dx.doi.org/10.1016/j.jtcvs.2013.07.070 trunk with endovascular completion was shown to be safe. $^{7,8}$

Making the decision between open or endovascular second-stage repair is still difficult. The objectives of the present study were to compare the acute morbidity and mortality and long-term survival of patients undergoing second-stage endovascular elephant trunk completion (EEC) or open elephant trunk completion (OEC) for extensive thoracic aortic disease.

\section{METHODS \\ Patients}

From August 1993 to November 2010, 225 of 405 patients who had undergone first-stage elephant trunk repair at the Cleveland Clinic underwent second-stage operations (Figure 1). Of these 225, $92(41 \%)$ were performed using endovascular stent grafts and $133(59 \%)$ were performed using conventional open techniques. The mean patient age was $63 \pm 12$ years (OEC, 60.8 years vs EEC, 67 years; range, 46-77). The data were obtained from the prospective cardiovascular information registry and chart review. The institutional review board of the Cleveland Clinic waived the requirement for informed consent and approved the use of the databases for the present study. Additional patient details are presented in Table 1.

\section{Treatment Selection and Timing}

We began performing EEC in 2000, and this technique has become increasingly common. For patients with degenerative aneurysms and an adequate distal landing zone, EEC has become our preferred option. 


\section{Abbreviations and Acronyms \\ $\mathrm{EC}=$ elephant trunk completion \\ $\mathrm{EEC}=$ endovascular elephant trunk completion \\ OEC $=$ open elephant trunk completion}

For patients with chronic aortic dissection and aneurysm, we initially offered EEC only to those at prohibitively high risk. ${ }^{8}$ In recent years, some moderate-risk patients with chronic dissection have been treated with EEC by modifying the distal aorta with an open fenestration. ${ }^{9}$ Nonetheless, a greater proportion of open patients had aortic dissection $(62 \%$ OEC vs $46 \%$ EEC, $P=.01$ ). To further assess the factors associated with deciding the approach, multivariate analysis was performed to develop a parsimonious model identifying the factors associated with EEC.

\section{First-Stage Elephant Trunk Procedure}

First-stage elephant trunk operations were performed using deep hypothermic circulatory arrest with or without brain perfusion. The arch anastomosis was performed either as a patch or with separate bypasses. The distal anastomosis was constructed either beyond or proximal to the left subclavian artery using an inversion of the graft in the distal aorta. ${ }^{10}$ As the first-stage procedure has become safer, the indications have expanded to patients with moderate distal dilatation as a prophylactic measure (Figure 1). All elephant trunk grafts were marked with large hemoclips for radiographic visualization should elephant trunk completion (EC) be performed.

\section{Second-Stage OEC}

All OECs were performed through a left posterolateral thoracotomy. Adjunctive cardiopulmonary bypass was used in most patients (98\%). Three patients underwent a clamp-and-sew technique for rupture, and one required circulatory arrest because of intraoperative bleeding from the arch. Intercostal artery reimplantation was performed for more extensive repairs when feasible (51\%). Most patients (89\%) had had a cerebrospinal fluid catheter inserted preoperatively. ${ }^{11,12}$ Additional operative details are listed in Table 1.

\section{Second-Stage EEC}

The endovascular procedures were performed in a hybrid suite with fixed imaging. Most patients had general anesthesia, but $28 \%$ had regional spinal anesthesia because of severe pulmonary disease. Access into the elephant trunk was obtained antegrade from the right brachial artery. The guidewire was snared from the common femoral artery to obtain through and through wire access. The stent graft was delivered by way of the iliofemoral system into the elephant trunk and deployed with an at least 2-stent overlap. Select patients requiring thoracoabdominal repair underwent second-stage repair using a custom-designed branched graft device as part of an investigational device exemption trial. ${ }^{13}$ Prophylactic cerebrospinal fluid drainage was performed preoperatively for most patients.

\section{Follow-up}

Cardiovascular information registry systematic follow-up information supplemented with the Social Security Death Index was used to determine vital status. The median follow-up was 3.7 years (mean \pm standard deviation, $4.0 \pm 3.1$ ). A total of 1620 patient-years was available for analysis. Of the survivors, $25 \%$ were followed up for $>6$ years and $10 \%$ for $>8$ years. For 4 patients, the date of the first-stage procedure was unknown, and they were excluded from analysis. Operative mortality data were available for all other patients. Late survival data were available for all EEC patients and $92 \%$ of OEC patients.
Of the eligible EEC patients, $93 \%$ were compliant, with $\geq 1$ imaging follow-up study available. Computed tomography was performed before discharge, within 6 months postoperatively, at 12 months postoperatively, and annually thereafter. Computed tomography of the chest, abdomen, and pelvis was performed without and with contrast enhancement, timed for the arterial and delayed venous phases, to assess for endoleaks. Computed tomography analysis was conducted using 3-dimensional techniques.

\section{Outcome Definitions and Statistical Analysis}

Stroke, paraplegia, and paraparesis were defined as a neurologic deficit lasting longer than 24 hours and confirmed by imaging and/or documentation by a neurologist. Spinal cord injury was further classified as immediate on waking from anesthesia or delayed after initially intact examination findings and as either persistent at discharge or transient if it had resolved before discharge. Renal failure was defined as the need for hemodialysis. Respiratory failure included reintubation or intensive care unit readmission for respiratory distress and included tracheostomy. Bleeding was defined by the need for reoperation.

Simple descriptive statistics were used to summarize the data. Continuous variables are presented as the mean \pm standard deviation. Group comparisons for categorical variables were made using a chi-square or Fisher's exact test. Continuous variables were compared using the Wilcoxon rank-sum test. Uncertainty is expressed by confidence limits equivalent to \pm 1 standard error $(68 \%)$.

A parsimonious model augmented with additional preoperative factors was created to identify the factors associated with the endovascular group and was used to form the propensity model. The propensity score for each patient is the probability of undergoing an endovascular procedure during second-stage EC. The patients were matched according to the propensity score, which included patients across the spectrum.

The long-term time-to-event (death or second-stage procedure) was assessed nonparametrically using the Kaplan-Meier method and parametrically using a multiphase hazard model. The parametric model was used to resolve the phases of instantaneous risk of death (hazard function) and to estimate the shaping parameters. ${ }^{14}$ In addition, competing risks analysis between death or second-stage procedure (EEC or OEC) was performed.

\section{RESULTS}

\section{Choosing EEC Versus OEC and Timing of Intervention}

Older age and atrial fibrillation were more likely to be associated with undergoing EEC than OEC. The OEC patients had a greater preoperative history of stroke and had undergone first-stage repairs in an early period. Chronic obstructive pulmonary disease and peripheral vascular disease were more common in the OEC group on univariate analysis but not on multivariate analysis.

A significant difference was found in the timing of the second stage between the 2 groups $(P[\log$-rank $]=.0013)$. The EEC group appears to have been more likely to require reintervention before about 3 months. After that, OEC was more likely to be used for second-stage repair (Figure 3, B).

\section{Morbidity and Mortality}

Although we found no difference in the overall frequency of complications between the 2 groups, the OEC group experienced a greater need for transfusion, a longer length of stay, and required tracheostomy more often (Table 2). 


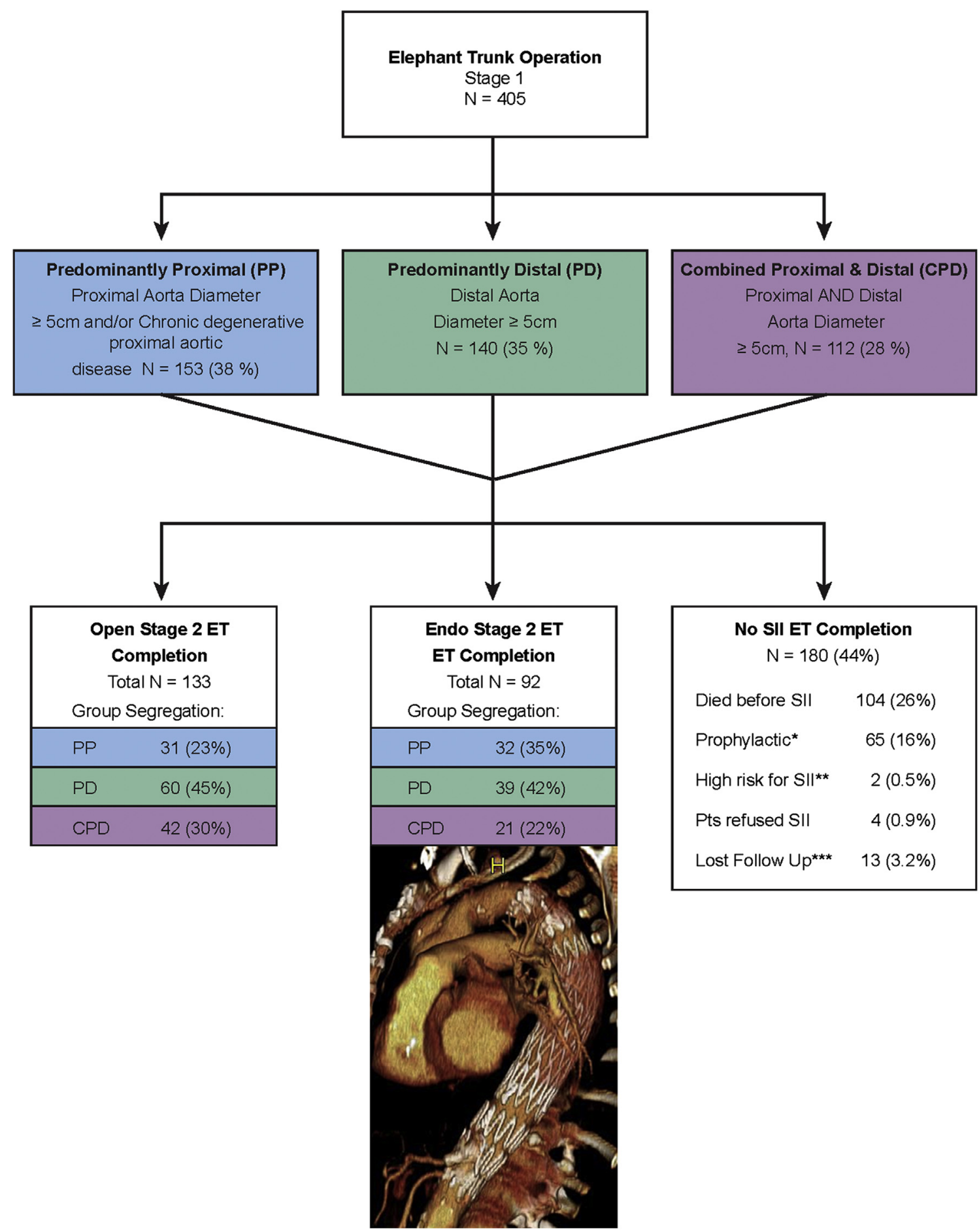

FIGURE 1. Schematic presentation of patient population with extensive aortic aneurysm disease undergoing elephant trunk reconstruction stratified by presentation. Volume-rendered computed tomographic reconstruction demonstrating the thoracic aorta after endovascular elephant trunk completion to the thoracoabdominal aorta. ET, Elephant trunk.

\section{Mortality}

The 30-day mortality was $6.2 \%$, and no difference was found between the EEC $(6.5 \%)$ and OEC $(6 \%)$ groups $(P=.884)$. In the propensity-matched comparison, no difference was found between the 2 groups. Of the OEC patients, 1 died intraoperatively of diffuse intravascular coagulopathy; 3 had severe intraoperative respiratory failure requiring extracorporeal membrane oxygenation 
TABLE 1. Patient characteristics

\begin{tabular}{|c|c|c|c|}
\hline Characteristic & OEC (n) & $\operatorname{EEC~(n)~}$ & $P$ value \\
\hline \multicolumn{4}{|l|}{ Preoperative } \\
\hline Age (y) & $60.8 \pm 12$ & $67 \pm 12.4$ & .0001 \\
\hline Preoperative BMI $\left(\mathrm{kg} / \mathrm{m}^{2}\right)$ & $26.5 \pm 5.04$ & $27 \pm 5.5$ & .075 \\
\hline Male gender & 90/133 (68) & $47 / 92(51)$ & .012 \\
\hline Hypertension & $107 / 122(88)$ & $77 / 73(84)$ & .4 \\
\hline Diabetes mellitus & $6 / 120(5)$ & 6/91 (7) & .43 \\
\hline History of stroke & 40/122 (33) & $6 / 92(6.5)$ & $<.0001$ \\
\hline Carotid disease & $61 / 122(50)$ & $29 / 89(33)$ & .012 \\
\hline History of MI & 29/122 (24) & 14/91 (15) & .13 \\
\hline Preoperative atrial dysrhythmias & $4 / 122(3.3)$ & 16/91 (18) & .0004 \\
\hline COPD & 48/121 (40) & 16/91 (18) & .0005 \\
\hline Smoking & 83/121 (69) & 68/88 (77) & .17 \\
\hline Chronic dissection & $81 / 130(62)$ & $42 / 92(46)$ & .014 \\
\hline Ascending repair before ET & $52 / 130(40)$ & $27 / 91(30)$ & .11 \\
\hline Emergency indication & 2/133 (1.5) & $6 / 91(6.6)$ & .044 \\
\hline Rupture & $2 / 130(1.5)$ & $4 / 92(4.3)$ & .2 \\
\hline \multicolumn{4}{|l|}{ Maximum aortic diameter } \\
\hline \multicolumn{4}{|l|}{ Before stage I } \\
\hline Proximal & $5.23 \pm 1.2$ & $4.99 \pm 1.3$ & .13 \\
\hline Distal & $5.94 \pm 1.2$ & $5.68 \pm 1.4$ & .088 \\
\hline \multicolumn{4}{|l|}{ Before stage II } \\
\hline Distal & $5.94 \pm 1.2$ & $5.8 \pm 0.8$ & .11 \\
\hline \multicolumn{4}{|l|}{ Operative details } \\
\hline \multicolumn{4}{|l|}{ Distal extent of repair } \\
\hline Descending & $89 / 126(71)$ & $53 / 91(58)$ & .058 \\
\hline TAAA & 37/126 (29) & $38 / 91(42)$ & .058 \\
\hline General anesthesia & $133 / 133(100)$ & $67 / 86(78)$ & $<.0001$ \\
\hline CSF drain & $84 / 89(94)$ & $48 / 59(81)$ & .13 \\
\hline Cardiopulmonary bypass & $128 / 130(98)$ & - & \\
\hline Left atrial-femoral artery & $84 / 109(77)$ & - & \\
\hline $\begin{array}{l}\text { Left femoral vein-femoral } \\
\text { artery }\end{array}$ & $8 / 109(7.3)$ & - & \\
\hline Clamp and sew & $3 / 130$ & - & \\
\hline DHCA & $10 / 130$ & - & \\
\hline Other & $2 / 128$ & - & \\
\hline Intercostals reimplanted & $55 / 119(46)$ & - & \\
\hline Stent graft type & - & & \\
\hline Homemade & - & $1 / 82(1.2)$ & \\
\hline TAG* & - & $18 / 82(22)$ & \\
\hline Talent $\dagger$ & - & $2 / 82(2.4)$ & \\
\hline Zenith $\ddagger$ & - & $61 / 82(74)$ & \\
\hline \multicolumn{4}{|l|}{ Stent grafts (n) } \\
\hline 1 & - & $24 / 92(26)$ & \\
\hline$>1$ & - & 68/92 (74) & \\
\hline Iliac conduit & - & $10 / 92(11)$ & \\
\hline
\end{tabular}

Data presented as mean \pm standard deviation or n $(\%)$. OEC, Open elephant trunk completion; $E E C$, endovascular elephant trunk completion; $B M I$, body mass index; $M I$, myocardial infarction; $C O P D$, chronic obstructive pulmonary disease; $E T$, elephant trunk; TAAA, thoracoabdominal aortic aneurysm; $C S F$, cerebrospinal fluid; $D H C A$, deep hypothermic circulatory arrest. *W. L. Gore \& Associates, Flagstaff, Ariz. $\dagger$ Medtronic, Minneapolis, Minn. $\ddagger$ Cook Medical, Bloomington, Ind.

support and died; 2 died of severe brain injury; and 2 died of unknown causes shortly after discharge, but both had residual dissections.

No EEC patients died intraoperatively. One died of rupture owing to retrograde pressurization of the false lumen after stent grafting of a chronic dissection. Five early EEC deaths were from septic complications. Two had undergone EEC for an aorto-esophageal fistula with sepsis, and neither survived. One had undergone EEC with fenestration of the dissection 3 days after first-stage elephant trunk because of acute distal malperfusion immediately after the first stage. This was complicated by sepsis, and the patient died. The fourth patient had an infected retroperitoneal hematoma, and the fifth died of pneumonia after discharge to a nursing facility.

\section{Bleeding}

No difference in bleeding was found between the 2 groups. The patients who bled after OEC required redo thoracotomy and decortication; 1 developed empyema. The bleeding complicating EEC was access related. Three patients required groin exploration for an expanding hematoma, and another required a thrombin injection for pseudoaneurysm. Three developed retroperitoneal bleeding related to an iliac conduit.

The OEC patients required transfusion of more red blood cells, plasma, and platelets than did the EEC patients, and the difference was maintained in the adjusted comparison (Table 2).

\section{Neurologic Complications}

Six patients experienced strokes (OEC, $\mathrm{n}=5$; EEC, $\mathrm{n}=1$ ). Of the 5 patients in the OEC group, the strokes led to brain death in 3,1 in a patient who required extracorporeal membrane oxygenation after myocardial infarction. One patient had dense hemiplegia, developed respiratory, and renal failure and died 4 months later in a nursing home. The fifth patient had seizures and dysphagia from a small infarct but had full resolution. The EEC patient experienced an embolic stroke from atrial fibrillation 2 days after discharge.

Finally, 2 OEC patients and 1 EEC patient had transient paraparesis, with full recovery. An additional 2 patients with paraparesis were discharged with residual weakness, and 2 OEC patients and 1 EEC patient were paralyzed.

\section{Respiratory Failure}

No difference was found in respiratory failure between the 2 groups, but 10 OEC patients required tracheostomy versus no EEC patients. Three early OEC deaths were from respiratory failure requiring extracorporeal membrane oxygenation.

\section{Renal Failure}

No difference was found in renal failure between the 2 groups. Two dialysis-dependent EEC patients died of septic complications during their hospitalization. Of the 6 OEC patients with renal failure, 2 recovered renal function and were weaned from dialysis and 4 required permanent dialysis. 

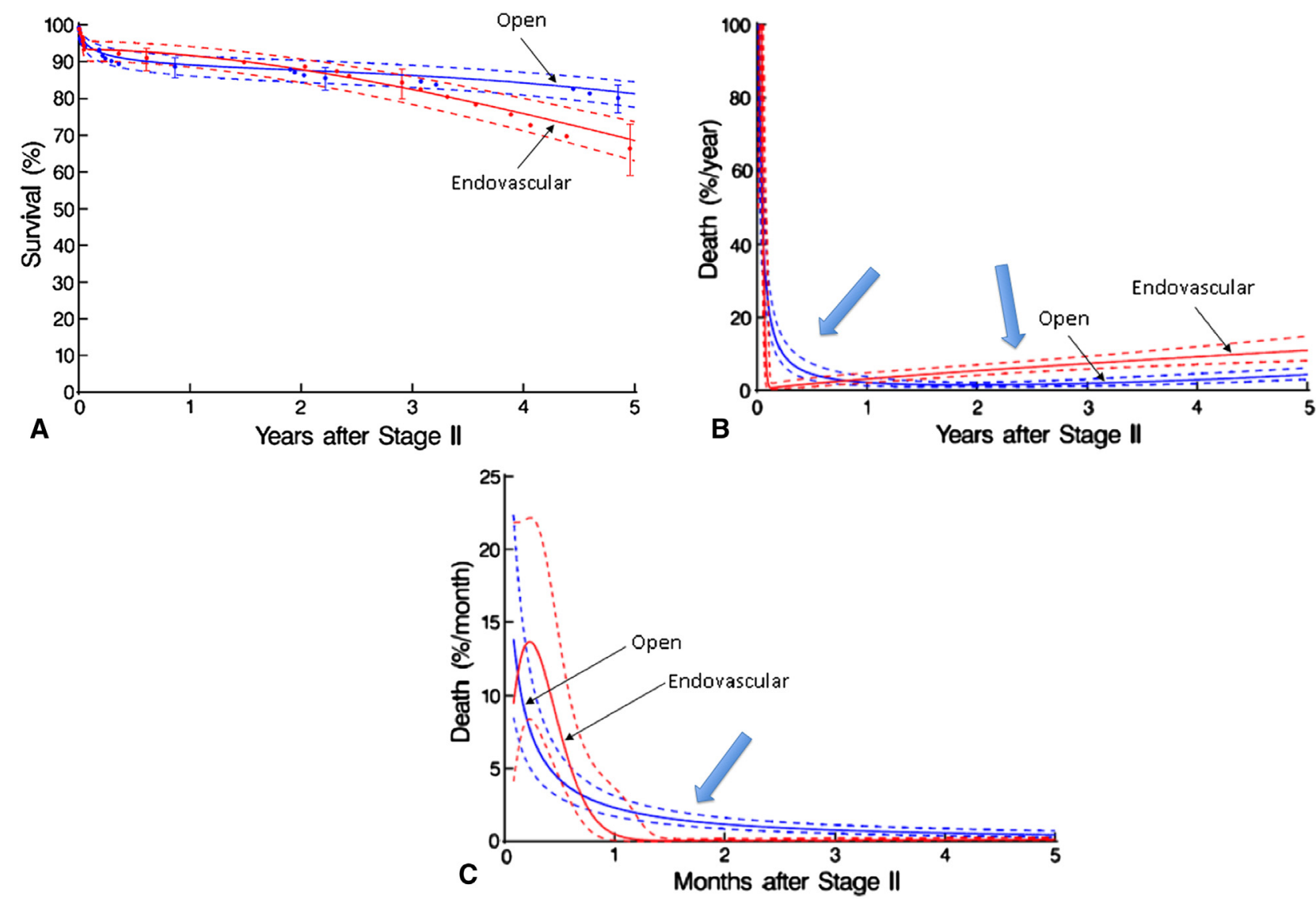

FIGURE 2. A, Survival after elephant trunk procedure stratified by open versus endovascular procedure. Each symbol represents a death positioned on a vertical axis the by Kaplan-Meier estimator. Vertical bars indicate confidence limits equivalent to 1 standard error. Solid lines indicate parametric survival estimates enclosed within dashed $68 \%$ confidence bands equivalent to 1 standard deviation. B, Instantaneous risk of death (hazard function) stratified by open versus endovascular procedures presented for 5 years. Note, both the early and later separation of curves (arrows). C, Detailed representation during first 6 months of instantaneous risk of death (hazard function) stratified by open versus endovascular procedures. This demonstrates the initial separation of curves seen in Figure $B$, and these curves separated after 1 month (arrow).

\section{Length of Stay}

The length of stay was longer for the OEC patients than for the EEC patients in both unadjusted and adjusted comparisons.

\section{Survival}

The overall parametric estimate of survival at 6 months and 1,3 , and 5 years was $91 \%, 90 \%, 85 \%$, and $77 \%$,
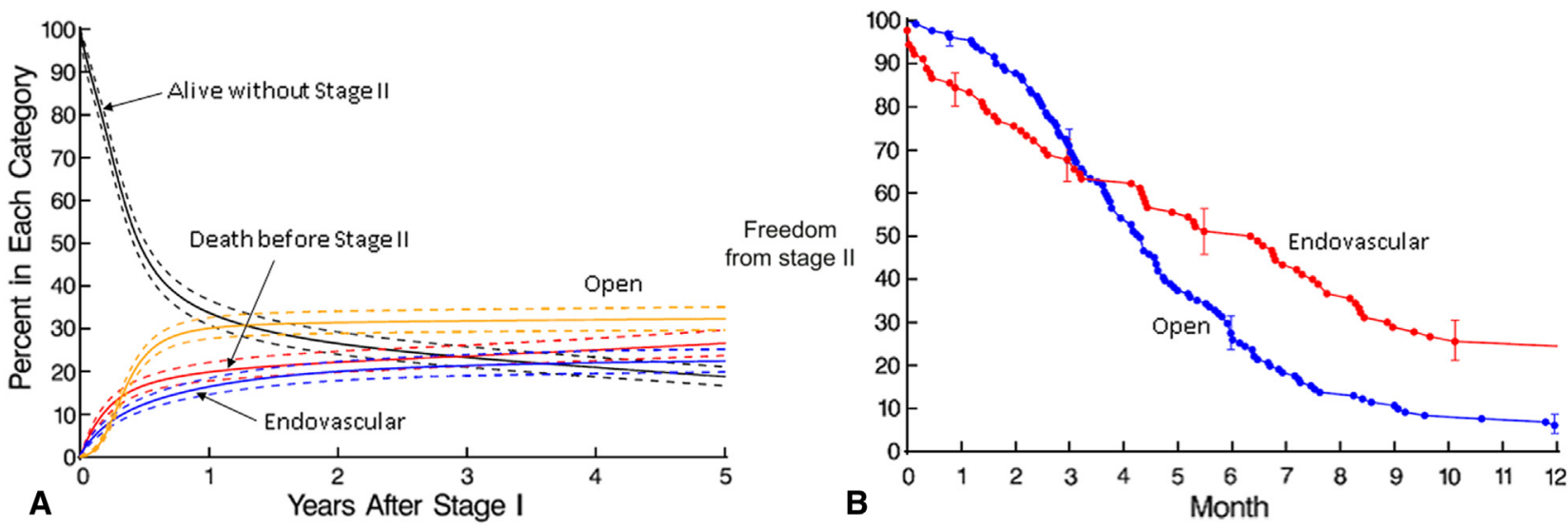

FIGURE 3. A, The competing risks of death, endovascular elephant trunk completion, and open elephant trunk completion. At each point, the sum of the 4 curves was $100 \%$. B, Interval to second-stage elephant trunk completion presented as nonparametric estimate of freedom from reintervention stratified by open versus endovascular procedure. 
TABLE 2. Morbidity and mortality

\begin{tabular}{|c|c|c|c|c|c|c|}
\hline \multirow[b]{2}{*}{ Event } & \multicolumn{2}{|c|}{ Overall population } & \multirow[b]{2}{*}{$P$ value } & \multicolumn{2}{|c|}{ Matched patients } & \multirow[b]{2}{*}{$P$ value } \\
\hline & OEC & EEC & & OEC & EEC & \\
\hline 30-d Mortality & $8 / 133(6)$ & $6 / 92(6.5)$ & .64 & $4 / 44(9.1)$ & $3 / 44(6.8)$ & .69 \\
\hline Bleeding & $11 / 123(8.9)$ & 8/91 (8.8) & .97 & $6 / 44(14)$ & $4 / 43(9.3)$ & .53 \\
\hline Stroke & $6 / 122(4.9)$ & $1 / 90(1.1)$ & .57 & $4 / 44(9.1)$ & $0 / 43(0)$ & .043 \\
\hline Respiratory failure & $15 / 122(12)$ & $6 / 90(6.7)$ & .18 & $7 / 44(16)$ & $2 / 43(4.7)$ & .085 \\
\hline Tracheostomy & $10 / 111(9)$ & $1 / 90(1.1)$ & .014 & $3 / 36(8.3)$ & $0 / 43(0)$ & .054 \\
\hline Renal failure & $6 / 122(4.9)$ & $3 / 90(3.3)$ & .57 & $2 / 44(4.5)$ & $2 / 43(4.7)$ & .98 \\
\hline Paraparesis & $5 / 132(3.8)$ & $4 / 92(4.3)$ & .83 & $1 / 43(2.3)$ & $3 / 44(6.8)$ & .32 \\
\hline Length of stay (d) & $14.9 \pm 10.6$ & $12.9 \pm 10.7$ & $<.04$ & $15 \pm 12.7$ & $9.36 \pm 10$ & .0005 \\
\hline \multicolumn{7}{|l|}{ Transfusion (U) } \\
\hline $\mathrm{RBCs}$ & $6.2 \pm 8.37$ & $3.21 \pm 7.75$ & .0001 & $6.41 \pm 8.68$ & $3.48 \pm 7.43$ & .0083 \\
\hline FFP & $5.41 \pm 6.59$ & $0.87 \pm 3.12$ & $<.0001$ & $4.55 \pm 5.45$ & $1.21 \pm 3.18$ & $<.0001$ \\
\hline Platelets & $4.17 \pm 6.2$ & $0.92 \pm 3.33$ & $<.0001$ & $3.16 \pm 5.16$ & $0.93 \pm 2.33$ & $<.0001$ \\
\hline
\end{tabular}

Data presented as n (\%). OEC, Open elephant trunk completion; EEC, endovascular elephant trunk completion; RBCs, red blood cells; $F F P$, fresh frozen plasma.

respectively (Figure 2, A). No significant difference was found in survival among the 2 groups in the overall or matched comparisons. Trends were seen toward better intermediate (6 months, $93 \%$ vs $86 \%$; 1 year, $90 \%$ vs $84 \%$, respectively) survival after EEC and better late survival after OEC.

The hazard function analysis demonstrated a difference in the instantaneous risk of death in the first 6 months (Figure 2, $B$ and $C$ ). The more focused study of hazard function within this period suggested a high early risk of death after EEC that diminished after the first 6 weeks. In contrast, the instantaneous risk of death decreased more slowly during the first 6 months in the OEC group (Figure 2,C). After the first 6 months, the slope to the hazard function was more constant for both groups.

\section{Intermediate Cause of Death}

A closer look at the cause of death was instructive. Between 30 days and 1 year, only 1 patient in the EEC group and 7 in the OEC group died. The EEC patient died of acute mesenteric ischemia from embolization of a thrombus near the proximal anastomosis. This patient weighed $>400 \mathrm{lb}$ and had undergone a multistage hybrid operation that included visceral and left renal debranching from the iliac artery before endovascular completion for chronic dissection. Of the 7 OEC patients, 5 died within months of discharge to a skilled nursing facility for severe perioperative complications. The sixth patient died of aortic rupture in the emergency department secondary to new distal dissection beyond the repair, and the seventh, with in-home nursing care, died of uncertain causes but had a known $5.8-\mathrm{cm}$ abdominal aneurysm that was scheduled for endovascular repair.

\section{Endoleaks and Reoperations}

Details regarding endoleaks and reoperations are listed in Table 3.
Endoleaks occurred frequently but most required no intervention. Of the 32 endoleaks, 4 required stent graft extensions, 7 patients underwent left subclavian embolization for type 2 endoleaks, and 1 underwent open repair for a distal type 1 endoleak.

Other vascular reoperations included 2 unplanned carotid to subclavian artery bypasses, 4 procedures for access complications, and 2 later stent grafts with balloon dilatation because of kinking of the elephant trunk. Two patients developed late pseudoaneurysms at the site of initial elephant trunk anastomosis that were easily treated with proximal extension stent grafts. Remote to the treated sites, 1 patient underwent root replacement, 1 had a left renal stent placed, and 1 had stenting of an acute superficial femoral dissection.

\section{Follow-up}

Regular follow-up data were less complete for the OEC patients because of long-distance referrals and a large proportion having undergone repair $>10$ years earlier. Of 85 patients with reliable follow-up data, $13(15.3 \%)$ required additional vascular reoperations. These included 2 proximal to the treated aorta: 1 redo coronary bypass grafting and 1 patient who had a left ventricular assist device placed followed by explantation and transplantation. Four OEC patients underwent procedures on the treated segment;

TABLE 3. Endoleaks and reoperations

\begin{tabular}{lcc}
\hline \multicolumn{1}{c}{ Event } & OEC & EEC \\
\hline Endoleak & - & $32 / 92(35)$ \\
I & - & $3 / 92(3)$ \\
II & - & $27 / 92(29)$ \\
III & - & $2 / 92(2.1)$ \\
Vascular reoperations & $13 / 85(15)$ & $26 / 92(28)$ \\
Treated segment & $4 / 85(5)$ & $16 / 92(17)$ \\
Proximal to treated segment & $2 / 85(2.3)$ & $3 / 92(3)$ \\
Distal to treated segment & $7 / 85(8)$ & $7 / 92(7.6)$ \\
\hline
\end{tabular}

Data presented as number of patients, with percentages in parentheses. OEC, Open elephant trunk completion; $E E C$, endovascular elephant trunk completion. 
all were done endovascularly: 2 pseudoaneurysms, 1 kinked graft, and 1 aneurysm of an intercostal patch. Seven required distal reoperations, six for progressive aneurysm and one for leg malperfusion. Of the 7 reoperations, 2 were done endovascularly with branched stent grafts, 2 with hybrid debranching techniques, and 3 open.

\section{DISCUSSION \\ Principal Findings}

The present report describes our multidisciplinary approach to patients with extensive aortic disease for various indications using the elephant trunk method. In the present comparison between OEC and EEC, we have demonstrated that equivalent results are achievable when the approaches are used as complementary strategies. Treatment of extensive aortic disease requires flexibility in decision-making because a patient's risk or morphology can change between stages. Careful evaluation by a multidisciplinary team allows for a fair assessment of the options to select the best strategy for each patient.

Given the complexity of this disease, patients treated with either approach can experience serious complications and are at late risk of reintervention. When serious complications occurred after OEC, they tended to be more disabling; however, the EEC group was more likely to require additional interventions. The OEC patients might experience more morbidity up front, requiring more blood transfusions and a longer hospital stay, but in the long term, their repairs might be more durable. Regardless of the technique used for elephant trunk completion, every patient requires lifelong follow-up with an aortic specialist.

\section{Different Populations}

OEC patients had more chronic obstructive pulmonary disease and history of stroke; however, the EEC patients tended to be older, with more atrial fibrillation and a greater requirement for emergency completion. We did not begin EEC until after 2000; thus, the OEC group included patients who had undergone first-stage repair during an earlier period. In the previous decade, first-stage operations have evolved, with nearly universal axillary cannulation and more antegrade brain perfusion. Both strategies have been shown to improve the neurologic outcomes in patients requiring circulatory arrest. The differences in surgical technique during the study period could explain the difference in stroke history between the cohorts and could also have played a role in the greater rate of perioperative stroke seen in OEC compared with EEC.

Because EEC is less traumatic than OEC, these patients were more likely to have undergone the second stage within the first 3 months after the first stage. In contrast, competing risks analysis demonstrated 2 peaks in the timing of OEC, with most occurring between 3 and 12 months (Figure 3).
This difference is important. Earlier completion should provide earlier protection from rupture. In previous elephant trunk repair series, the risk of death between stages attributable to incomplete repair has been consistent. ${ }^{20}$ Some patients will experience aortic complications very early after first-stage repair because of technical issues related to the complexity of arch repair. The rescue strategy for these patients has been emergency stent grafting, because endovascular reperfusion can be accomplished more expeditiously in a hybrid operating room than emergency OEC. Although emergency EEC is feasible, the emergency indications for distal aortic surgery still carry a high risk. Three patients who underwent EEC for unexpected emergency indications died, and their deaths might have skewed the results out of favor for EEC.

Nonetheless, many EEC patients would not have been able to undergo an open operation and benefitted from the endovascular option. This population includes elderly patients with degenerative aneurysms who often have chronic obstructive pulmonary disease and vascular occlusive disease and have an increased risk of neurologic complications.

\section{Patient Selection}

Few patients were treated with a simple open anastomosis of the distal elephant trunk or the use of a single stent graft device. More typically, patients required more extensive reconstruction. In our institution, the most important determinants for an open or endovascular approach are the disease etiology and extent and the patient's comorbid risk profile.

Patients with degenerative disease typically undergo EEC if their distal anatomy is conducive to stent grafting. With the addition of branched stent grafts, most patients with degenerative etiology can safely undergo EEC. ${ }^{21} \mathrm{~A}$ small subset of young patients with degenerative thoracoabdominal aneurysms will still require OEC because of anatomic and durability considerations. Many patients with chronic aortic dissection will undergo OEC because endovascular treatment of this morphology is less reliable. To facilitate a distal seal when stent grafting extensive chronic dissection, we introduced adjunctive fenestration at first-stage elephant trunk repair, with excellent results. ${ }^{22}$ As branched grafts continue to improve, we could see more patients undergoing endovascular procedures.

From a neurologic risk perspective, we have previously shown that no difference in outcome exists between open and endovascular repair in patients matched across the extent of repair, ${ }^{17}$ and that was confirmed again in the present study.

EEC techniques can expedite second-stage repair because patients still recovering from the first stage can usually tolerate EEC. This is important for patients with severe disease of both the proximal and the distal thoracic 
aorta. The exception is when a distal seal requires the use of a branched stent graft. These devices are currently only available as part of an investigational device exemption, and each patient's device requires several weeks for customized construction.

We have selectively used hybrid debranching techniques to facilitate earlier second-stage repair with readily available devices. ${ }^{23}$ Although the mortality has been low using single-stage repair through a bilateral anterior thoracotomy (ie, clamshell) incision, the morbidity has been high with a need for tracheostomy in $13 \%$. These results were similar to those we found in the OEC group; however, the interval mortality was eliminated with the single-stage strategy. ${ }^{17}$ Another option for patients who cannot tolerate a delay between proximal and distal repair is to perform their repair in a hybrid single-stage fashion using antegrade stent graft delivery techniques (ie, frozen elephant trunk). ${ }^{19,24}$ The frozen elephant trunk technique could provide a better option for the subset of patients who experienced early perioperative complications requiring emergency EEC. A better understanding of how to select those patients is needed.

With OEC, all morphologies are treatable, and the repair can easily extend to the pelvic vasculature. More extensive repair, however, requires a lot from patients and could partially explain the high rate of long-term respiratory failure seen after OEC. We offer OEC to lower risk patients with thoracoabdominal aneurysms in the setting of chronic dissection and to the lowest risk patients with aneurysm limited to the descending aorta.

Patients with connective tissue disorders, such as Marfan syndrome, warrant additional discussion. These patients have a high lifetime risk of multiple vascular reoperations. ${ }^{25,26}$ When feasible, we have relied on open surgical repair techniques to treat these patients because the durability of a stent graft repair is dependent on the integrity of the vessel where it is placed. EEC has been reserved for the highest risk patients in this subgroup; however, the graft material of the elephant trunk provides a reliable landing zone for stent grafting. Most patients with connective tissue disorders who underwent EEC presented with an urgent indication. Although many patients have subsequently required additional procedures, the proximal end of the stent graft has remained well fixed within the surgical graft, with no migrations.

\section{Completeness of Repair}

Any patient with extensive aortic disease is at risk of late vascular reoperations. This is true, regardless of the etiology or treatment strategy. Even patients with entirely prosthetic aortas have required additional procedures. Thus, we believe it is paramount that all patients with extensive aortic disease be followed up with regular cross-sectional imaging studies by an aortic disease specialist.

\section{Study Limitations}

Even with propensity matching, it can be difficult in a diverse group of this size to account for all selection bias. The outcomes with both OEC and EEC have been good; thus, there were few events to assess. Until more experience and data are accrued, it will be difficult to demonstrate the superiority of 1 technique over another within any subgroup. Late clinical and imaging follow-up in many OEC patients was limited by the need for travel. Only more recently have we implemented stricter protocols for radiographic follow-up.

\section{CONCLUSIONS}

Death and complications occurred similarly after OEC or EEC. The early toll on patients might be greater after OEC, at the cost of reintervention for EEC. EEC expands the options to older patients and allows for earlier completion. Second-stage repair should not be delayed, and all patients require lifelong imaging surveillance.

\section{References}

1. Svensson LG, Kim KH, Blackstone EH, Alster JM, McCarthy PM, Greenberg RK, et al. Elephant trunk procedure: newer indications and uses. Ann Thorac Surg. 2004;78:109-16.

2. Schepens MA, Dossche KM, Morshuis WJ, van den Barselaar PJ, Heijmen RH, Vermeulen FE. The elephant trunk technique: operative results in 100 consecutive patients. Eur J Cardiothorac Surg. 2002;21:276-81.

3. LeMaire SA, Carter SA, Coselli JS. The elephant trunk technique for staged repair of complex aneurysms of the entire thoracic aorta. Ann Thorac Surg. 2006;81:1561-9

4. Safi HJ, Miller CC, Estrera AL, Villa MA, Goodrick JS, Porat E, et al Optimization of aortic arch replacement: two-stage approach. Ann Thorac Surg. 2007;83(suppl):S815-8

5. Etz CD, Plestis KA, Kari FA, Luehr M, Bodian CA, Spielvogel D, et al. Staged repair of thoracic and thoracoabdominal aortic aneurysms using the elephan trunk technique: a consecutive series of 215 first stage and 120 complete repairs. Eur J Cardiothorac Surg. 2008;34:605-15.

6. Fann JI, Dake MD, Semba CP, Liddell RP, Pfeffer TA, Miller DC. Endovascular stent-grafting after arch aneurysm repair using the "elephant trunk" Ann Thorac Surg. 1995;60:1102-5

7. Greenberg RK, Haddad F, Svensson L, O'Neill S, Walker E, Lyden SP, et al. Hybrid approaches to thoracic aortic aneurysms: the role of endovascular elephant trunk completion. Circulation. 2005;112:2619-26.

8. Kang WC, Greenberg RK, Mastracci TM, Eagleton MJ, Hernandez AV, Pujara AC, et al. Endovascular repair of complicated chronic distal aortic dissections: intermediate outcomes and complications. J Thorac Cardiovasc Surg. 2011;142:1074-83

9. Roselli EE, Sepulveda E, Pujara AC, Idrees J, Nowicki E. Distal landing zone open fenestration facilitates endovascular elephant trunk completion and false lumen thrombosis for extensive chronic dissection. Ann Thorac Surg. 2011;92:2078-84

10. Svensson LG. Rationale and technique for replacement of the ascending aorta, arch, and distal aorta using a modified elephant trunk procedure. J Card Surg. 1992; 7:301-12.

11. Lima B, Nowicki ER, Blackstone EH, Williams SJ, Roselli EE, Sabik JF III, et al. Spinal cord protective strategies during descending and thoracoabdominal aortic aneurysm repair in the modern era: the role of intrathecal papaverine. J Thorac Cardiovasc Surg. 2012;143:945-52.

12. Safi HJ, Miller CC III, Huynh TT, Estrera AL, Porat EE, Winnerkvist AN, et al Distal aortic perfusion and cerebrospinal fluid drainage for thoracoabdominal and descending thoracic aortic repair: ten years of organ protection. Ann Surg. 2003;238:372-80.

13. Roselli EE, Greenberg RK, Pfaff K, Francis C, Svensson LG, Lytle BW Endovascular treatment of thoracoabdominal aortic aneurysms. J Thorac Cardiovasc Surg. 2007;133:1474-82. 
14. Blackstone EH, Naftel DC, Turner ME Jr. The decomposition of time-varying hazard into phases, each incorporating a separate stream of concomitant information. J Am Stat Assoc. 1986;81:615-24.

15. Svensson LG, Blackstone EH, Rajeswaran J, Sabik JF III, Lytle BW, GonzalezStawinski G, et al. Does the arterial cannulation site for circulatory arrest influence stroke risk? Ann Thorac Surg. 2004:78:1274-84; discussion 1274-84.

16. Greenberg RK, Lu Q, Roselli EE, Svensson LG, Moon MC, Hernandez AV, et al. Contemporary analysis of descending thoracic and thoracoabdominal aneurysm repair: a comparison of endovascular and open techniques. Circulation. 2008; 118:808-17.

17. Kouchoukos NT, Mauney MC, Masetti P, Castner C. Optimization of aortic arch replacement with a one-stage approach. Ann Thorac Surg. 2007;83: S811-4.

18. Roselli EE, Soltesz EG, Mastracci T, Svensson LG, Lytle BW. Antegrade delivery of stent grafts to treat complex thoracic aortic disease. Ann Thorac Surg. 2010;90:539-46.

19. Lima B, Roselli EE, Soltesz EG, Johnston DR, Pujara AC, Idrees J, et al. Modified and "reverse" frozen elephant trunk repairs for extensive disease and complications after stent grafting. Ann Thorac Surg. 2012;93:103-9; discussion 109

20. Svensson LG, Rushing GD, Valenzuela ES, Rafael AE, Batizy LH, Blackstone EH, et al. Modifications, classification, and outcomes of elephant trunk procedures. Ann Thorac Surg. 2013;96:548-58.

21. Greenberg R, Eagleton M, Mastracci T. Branched endografts for thoracoabdominal aneurysms. J Thorac Cardiovasc Surg. 2010;140(6 suppl):S171-8.

22. Roselli EE, Sepulveda E, Pujara AC, Idrees J, Nowicki E. Distal landing zone open fenestration facilitates endovascular elephant trunk completion and false lumen thrombosis. Ann Thorac Surg. 2011;92:2078-84.

23. Vivacqua A, Albacker TB, Roselli EE. Hybrid thoracoabdominal aneurysm repair with antegrade visceral debranching from the ascending aorta: concomitant cardiac surgery and stent-grafting. Ann Thorac Surg. 2011;92: 2275-7.

24. Idrees J, Keshavamurthy S, Subramanian S, Clair DG, Svensson LG, Roselli EE. Hybrid repair of Kommerell diverticulum. J Thorac Cardiovasc Surg. March 24, 2013 [Epub ahead of print].

25. Svensson LG, Kouchoukos NT, Miller DC, Bavaria JE, Coselli JS, Curi MA, et al. Expert consensus document on the treatment of descending thoracic aortic disease using endovascular stent-grafts. Ann Thorac Surg. 2008;85(1 suppl): S1-41.

26. Subramanian S, Roselli EE. Thoracic aortic dissection: long-term results of endovascular and open repair. Semin Vasc Surg. 2009;22:61-8.

\section{Discussion}

Dr D. Craig Miller (Stanford, Calif). Thank you, Dr Bremner. I would like all of you to keep your eyes on Eric Roselli, who is a potential superstar in our field in the future. Not only is he trained interventionally but he is also open-surgical trained. He can do a transfemoral or transapical percutaneous aortic valve replacement in the morning and then an open aortic valve replacement in the afternoon. He can do a thoracic endovascular aortic repair (TEVAR) with the best of them, as well as open thoracoabdominal or descending thoracic aortic aneurysm repairs. In my book, Eric and others of his ilk represent the future for cardiovascular surgery. I want to compliment him and the Cleveland aortic surgery group for a clear analysis of a complicated group of patients. This work was superbly presented.

Before we dig into the meat of this presentation, which actually is pretty straightforward, there is an overarching general question that I would like to ask. Of 353 patients who underwent a firststage arch replacement procedure with the elephant trunk, 156 were not accounted for in this analysis. They had died at the first operation, they had died postoperatively, or they came back and were either deemed not fit for a second-stage elephant trunk completion procedure or declined it. Thus, you have this large decrease in patient numbers from the initial arch operation to the second-stage completion procedure. If you consider something similar to a $5 \%$ to $10 \%$ operative risk for the initial arch replacement, a $20 \%$ attrition rate in the interval between procedures, and then another $5 \%$ to $10 \%$ or so death risk for the second-stage elephant trunk completion procedure, that adds up to some serious numbers. This excludes, of course, an unknown fraction in which the descending aortic aneurysm or dissection does not enlarge. Therefore, should we rethink this whole concept? Should we do what Nick Kouchoukos has advocated for years: the arch-first approach, in which one does the arch and descending thoracic or thoracoabdominal aortic aneurysm aortic graft replacement simultaneously at 1 operation? In his most recent 2008 Annals of Thoracic Surgery report of 51 patients with extensive chronic aortic dissections, Dr Kouchoukos had a $4 \%$ operative mortality rate, a $2 \%$ incidence of paraplegia, a 75\% 5-year survival rate, and a $90 \%$ estimate of freedom from reoperation at 7 years. We all would agree that Dr Kouchoukos' medium-term results are truly excellent. About $56 \%$ of the patients in your series had a chronic dissection and tended to be treated with an open surgical second-stage procedure instead of endovascular completion. So, would 1 large operation and, in Nick's hands a safe one, be a better alternative strategy to the 2-stage approach you reported today, regardless of how the second stage is conducted? The downside of Nick's operation using a thoracosternotomy or bilateral sternotomy is major morbidity (eg, $18 \%$ required a tracheostomy), so there certainly is a price to be paid. As with everything in life, you either pay now or you pay later. What do you think, Eric, about the larger overall picture? Should we give more thought to doing these extensive thoracic aortic and thoracoabdominal procedures in 1 stage?

Dr Roselli. Well, first of all, thanks for the kind comments. This has been a really exciting experience treating these patients and using these new technologies.

What happens to these patients after the first-stage operation is a question I cannot answer completely at this time. Right now, it looks like we have done nearly 500 first-stage elephant trunk procedures at our institution, and we are in the process of collecting the data because we do have a better practice of keeping track of these folks. Many of these patients have undergone a first-stage elephant trunk operation as a prophylactic measure. For example, a patient with a 48-mm descending aorta is going to undergo an elephant trunk when we have to replace the arch, but there certainly are a certain percentage of patients who do die in the interim or do not want to return for a second stage. I can tell you from my own personal experience, whatever that is worth, that when I do talk to the patients about having a stent graft up front and the plan is to get the thing completed, they do come back. I think that will make a big difference, but we are in the process of combing through that data to have a better sense of it.

As far as the second comment about doing it all in 1 stage, I think that also makes a lot of sense, and, you are right, it is still a pretty morbid operation, even in Dr Kouchoukos' excellent experience. Other options exist to do it in a first stage, and we do the clamshell operation in Cleveland once in a while. My preference if I have to perform an operation in 1 stage because the proximal and distal aorta are both very large, however, is to use what I 
term a "frozen elephant trunk operation," and I will put the stent graft in through a sternotomy at the same time as the proximal repair. We have had pretty good results in that group of patients as well. We did not include any of those in this analysis, which included only the 2-stage operations. However, I think as this technology improves and our understanding of how to treat these patients improves, we can tailor the approach. The clamshell operation will still have a role, but I would not want to use it in somebody with bad lungs. I would rather do a frozen elephant trunk.

Dr Miller. Okay, good response. I would be interested in what happened to those other patients. I am sure the patients are more interested in returning if you promise them a stent graft rather than a thoracotomy.

That gives rise to my second question. What is the big deal about a second-stage completion procedure, no matter how you do it? It is really not a high-risk procedure, as you have shown today. The operative mortality and morbidity rates are low. In fact, you were surprised that no difference was found in the early results between the endovascular and open approaches, although the TEVAR endovascular patients were older and sicker. In your propensity score-adjusted matched cohorts, you could not show that 1 approach was better than the other. Therefore, why would you use a more palliative TEVAR procedure, instead of a much more durable operation if no difference exists in the upfront risk?

Dr Roselli. It might be blasphemy for me to say so, but I do not think the statistics can actually show us some of the finer details and differences in these groups of patients, because the numbers are just too small. There are clearly patients who have undergone these operations as rescue attempts with these stent grafts for whom we would never even think about doing an open operation. This group of patients who we treated consists of patients who we have considered with the broad eye of having all the treatment options in our armamentarium. The open operation is good, the stent graft is good, and there are patients who we just probably should not treat at all, and we are still trying to determine which patients belong in that last group.

Dr Miller. Good for you; you smelled my next question coming. You do have the opportunity, the expertise, and the results to prove you can use whichever method is best for that patient, and I commend you for that. Why does not the rest of the world have that capability? Are we "dumbing down" the entire field of thoracic aortic disease treatment because many, if not most, centers cannot perform open thoracic aortic surgical repairs with acceptable results or will not refer the patients to high-volume regional referral surgical centers that can produce good results? Instead, they insist on doing a hybrid arch or hybrid thoracoabdominal TEVAR compromise procedure, which has not been associated with any less early risk and has limited short-term durability. Are we "dumbing down" the entire field today?

Dr Roselli. That is a tough question to answer. To this audience, I would say that is not a problem. I think there are cardiac surgeons who call us all the time to send us these patients, understanding that they need to be evaluated at a tertiary center that does a large number of these operations. However, there certainly are places where surgeons do come up with these unproven methods of treating patients. I know, for example, that at some of the vascular surgical meetings I have listened to discussions of performing arch operations with a femoral to subclavian to carotid to carotid bypass before putting a stent graft in. That makes no sense to me, to use a leg-to-brain bypass in somebody before fixing their aorta when we have very good proven techniques, but it happens. I do not know how to fix that.

Dr Miller. Well, like it or not, it will probably be the payors who eventually are going to address this dilemma for us, and it will not just happen in the United Kingdom. I predict there will be a US equivalent of the British "NICE" committee, which will measure cost effectiveness by calculating the "qualy's" (quality years of survival gained) against the cumulative cost and will then determine whether hospitals and physicians will be paid for a procedure. That is where we will be forced to spend our limited resources. This delicate point relates to my final question: Philippe Demers at this meeting in 2003 presented the mid-term outcomes for our first 103 thoracic aortic stent graft TEVAR patients from Stanford. Two thirds of the patients had been deemed inoperable by Scott Mitchell or myself, and the 5-year survival rate after TEVAR was dismally low-31\% $\pm 6 \%$-for this cohort, because, as one might have expected, they mostly died of complications from their multiple medical comorbidities even if aneurysm rupture was prevented. This led us to conclude on philosophical grounds that TEVAR should not be performed in asymptomatic patients who were judged by a cardiovascular surgeon not to be fit enough to undergo open surgical graft replacement (J Thorac Cardiovasc Surg. 2004;127:664-73). No matter how one assesses the "qualy's" for these unfortunate patients, TEVAR just does not compute. Eric, what are the sentiments of you and your colleagues concerning how we address these issues about the allocation of fixed US healthcare resources in the future?

Dr Roselli. Again, that is another difficult problem. We see it not only with patients with thoracic aortic disease who have a limited lifespan no matter what we do, but also we are seeing it more and more in these groups of patients with severe aortic stenosis who are being evaluated for transcatheter valve replacement. How do you define the highest risk group of patients? I think we need to just ensure that we collect the data on all these patients we treat and the ones we do not treat to have a better understanding of what happens.

Dr Miller. Again, a great presentation and welcome to the Western Thoracic.

Dr Roselli. Thank you.

Dr Richard Lee (Chicago, Ill). Eric, again congratulations on this contribution. I think your data, regardless of the statistical analysis, do suggest that for the early mortality or near early mortality, the procedure itself caused a lot of mortality in the open group. For the late mortality, it evens out. Any insight into the cause of mortality in the late group and are there any mortalities due to the endovascular repair in the late group?

Dr Roselli. Great question, Rick. Thanks. So far, the late deaths that I have been able to determine the cause of have been because of various etiologies. Several patients died of cancer. I have seen 1 patient die of aortic disease-a patient who would not return for follow-up - who died of an abdominal aneurysm that was scheduled to be repaired but ruptured. Another patient died of rupture of a chronic dissection. However, most of them have been agerelated illnesses, less so aortic-related deaths, from what we can tell at this point. 